\title{
AN EXAMPLE OF A SPACE WHICH IS COUNTABLY COMPACT WHOSE SQUARE IS COUNTABLY PARACOMPACT BUT NOT COUNTABLY COMPACT
}

\author{
LEE PARSONS
}

\begin{abstract}
A subspace $P$ of $\beta N-N$ is obtained whose square is disjoint from the graph, $G$, of a pre-selected homeomorphism $f: \beta N \rightarrow \beta N$ that has no fixed points. The construction is performed in such a way that, for $X=P \cup N$, all countable subsets of $X^{2}-G$ will have a limit point in $X^{2}$. We use the following lemma: If $K \subset(\beta N)^{2}-G$ is countably infinite, then $\left|\mathrm{cl}_{(\beta N)^{2}} K-G\right|=2^{c}$.
\end{abstract}

We construct the space $X$ using the technique of J. Novák [N]. In the reference cited, Novák constructs a countably compact space whose square is not countably compact. Several versions have appeared in the literature. $\mathbf{H}$. Terasaka's example [T] is presented in Gillman and Jerison, Rings of continuous functions [GJ] and in Steen and Seebach, Counterexamples in topology [SS]. Novák's example was modified by Frolík in [F]. The latter version is presented by Engelking in [E], Outline of general topology.

A subspace $P$ of $\beta N-N$ will be obtained whose square is disjoint from the graph, $G$, of a preselected homeomorphism $f: \beta N \rightarrow \beta N$ that has no fixed point, but has the property that $f^{2}=f$. The notation of [GJ] is used, primarily. The construction will be performed in such a way that all countable subsets of $X^{2}-G$ will have a limit point in $X^{2}$, where $X=P \cup N$. Then $X$ will be countably compact since it is homeomorphic to a closed subset of $X^{2}-G$. Moreover, $G \cap X^{2}=\{(n, f(n)): n \in N\}$ is closed in $X^{2}$ and is an infinite discrete set, so $X^{2}$ is not countably compact. But $X^{2}=\left(X^{2}\right.$ $\cap G) \cup\left(X^{2}-G\right)$ is the disjoint union of a countably compact subspace and a countable, clopen discrete subspace and hence is countably paracompact.

The burden of proof is borne mostly by the following

LEMMA. If $K \subset(\beta N)^{2}-G$ is countably infinite then $\left|\mathrm{cl}_{(\beta N)}{ }^{2} K-G\right|=2^{2^{\omega}}$.

Proof. Suppose that $K \subset(\beta N)^{2}-G$ is countably infinite. We let $\pi_{1}$ and $\pi_{2}$ denote the projections onto the first and second factors of subsets of $(\beta N)^{2}$. If there is a point $p \in N$ such that $H=K \cap(\{p\} \times N)$ is infinite then $|\mathrm{cl} H| \geqslant\left|\mathrm{cl}\left(\pi_{2} H\right)\right|=2^{c}$, noting that $\mathrm{cl} \pi_{2} H=\pi_{2} \mathrm{cl} H$. But $\mid G \cap(\{p\} \times$

Presented to the Society, January 23, 1976; received by the editors November 7, 1975.

AMS (MOS) subject classifications (1970). Primary 54D20; Secondary 54G20.

Key words and phrases. Countably compact, countably paracompact, extremally disconnected, $M$-space, pseudocompact. 
$\beta N) \mid=1$ so that the lemma holds in this and the analogous case, $H-(\beta N \times$ $\{p\}) \cap K$.

We may now assume throughout (by choosing an appropriate infinite subset of $K$ ) that

$$
|K \cap(\{p\} \times \beta N)| \vee|K \cap(\beta N \times\{p\})| \leqslant 1
$$

for all $p \in \beta N$, and $\pi_{i} K$ is infinite, $\quad i=1,2$.

If $K^{\prime} \subset K$ is countably infinite and has the property that $\operatorname{cl}\left(f\left[\pi_{1}\left[K^{\prime}\right]\right]\right) \cap$

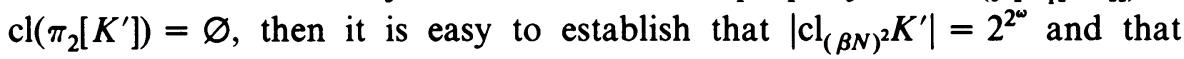

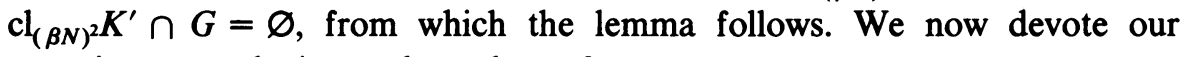
attention to producing such a subset of $K$.

Every countably infinite subset of $\beta N$ has a countably infinite subset whose topology inherited from $\beta N$ is discrete. Now, using this fact, choose an infinite subset $K^{*} \subset K$ such that $\pi_{1}\left[K^{*}\right]$ is discrete. Then $f\left[\pi_{1}\left[K^{*}\right]\right]$ is discrete, since $f$ is a homeomorphism. Apply this same technique again to obtain $K^{* *} \subset K^{*}$, countably infinite, such that $\pi_{2}\left[K^{* *}\right]$ is discrete. By assumption $(*), K^{* *}$ has the property that $f\left[\pi_{1}\left[K^{* *}\right]\right]$ and $\pi_{2}\left[K^{* *}\right]$ are infinite, discrete topological subspaces of $\beta N$. Since it is a bit tedious to carry the **'s about, let us assume without loss of generality that $K$ has the latter property to begin with.

Now cull $K$ again. Let $K$ be enumerated as $\left\{\left(p_{1}, q_{1}\right),\left(p_{2}, q_{2}\right), \ldots\right\}$. Let $i_{1}=1$. Let $U_{1}$ be a neighborhood of $q_{i_{1}}$ which misses $f\left(p_{i_{1}}\right)$ and infinitely many points of $f\left[\pi_{1}[K]\right]$ and whose intersection with $\pi_{2}[K]$ is $\left\{q_{i_{1}}\right\}$. Now suppose $i_{1}, \ldots, i_{n}$ are selected in such a way that $f\left[\pi_{1}[K]\right]-\cup_{i=1}^{n} U_{i}$ is infinite and $f\left(p_{i_{j}}\right) \notin \cup_{i=1}^{n} U_{n}$ for $j=1, \ldots, n$, and $\cup_{i=1}^{n} U_{i} \cap \pi_{2}[K]=$ $\left\{q_{i_{1}}, \ldots, q_{i_{n}}\right\}$. Now choose $i_{n+1}$ so that $f\left(p_{i_{n+1}}\right) \in f\left[\pi_{1}[K]\right]-\bigcup_{i=1}^{n} U_{i}$. Then choose $U_{n+1}$ so that, one, it does not contain $f\left(p_{i_{j}}\right), j=1, \ldots, n+1$; two, its intersection with $\pi_{2}[K]$ is $\left\{q_{i_{n+1}}\right\}$; and three, it misses infinitely many members of $f\left[\pi_{1}[K]\right]-\cup_{i=1}^{n} U_{i}$. The inductive selection of the sequence $\left\langle i_{1}, i_{2}, \ldots\right\rangle$ is complete. Denote by $K^{\prime \prime}$ the subset $\left\{\left(p_{i_{1}}, q_{i_{1}}\right),\left(p_{i_{2}}, q_{i_{2}}\right), \ldots\right\}$ of $K$. Then $\cup_{i=1}^{\infty} U_{i}$ is a neighborhood of $\pi_{2} K^{\prime \prime}$ and $\cup_{i=1}^{\infty} U_{i} \cap f\left[\pi_{1}\left[K^{\prime \prime}\right]\right]=\varnothing$. Thus $\mathrm{cl}_{\beta N} f\left[\pi_{1}\left[K^{\prime \prime}\right]\right] \cap \pi_{2} K^{\prime \prime}=\varnothing$. In an exactly analogous manner, we pick an infinite subset $K^{\prime} \subset K^{\prime \prime}$ having the property that $f\left[\pi_{1}\left[K^{\prime}\right]\right] \cap \mathrm{cl}\left(\pi_{2}\left[K^{\prime}\right]\right)=$ $\varnothing$. Then it follows that $\operatorname{cl} f\left[\pi_{1}\left[K^{\prime}\right]\right] \cap \operatorname{cl} \pi_{2}\left[K^{\prime}\right]=\varnothing$.

Note that $\mathrm{cl} \pi_{i} K^{\prime}=\pi_{i} \mathrm{cl} K^{\prime}, i=1,2$, so that we actually proved:

If $K \subset(\beta N)^{2}$ is countably infinite and if $\{p \in \beta N:(\beta N \times\{p\}) \cap K \neq$ $\varnothing\}$ and $\{p \in \beta N:(\{p\} \times \beta N) \cap K \neq \varnothing\}$ are infinite, then

$$
\begin{aligned}
\mid\{r \in \beta N: \exists s & \in \beta N,(r, s) \in \operatorname{cl} K-G\} \mid \\
& =|\{s \in \beta N: \exists r \in \beta N,(r, s) \in \operatorname{cl} K-G\}|=2^{c} .
\end{aligned}
$$

Now, beginning the construction of $X$, we index the countable subsets of

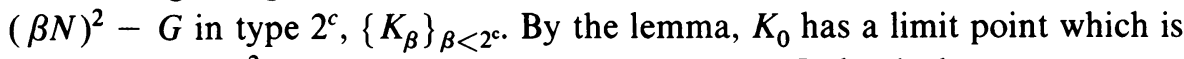
not in $G \cup N^{2},\left(r_{0}, s_{0}\right)$. Let $P_{0}=\left\{r_{0}, s_{0}\right\}-N$. Inductively, suppose $P_{\alpha}$, 
$\alpha<\beta$, are selected so that $P_{\alpha} \subset P_{\gamma}$ for $\alpha<\gamma<\beta$ and $f\left[P_{\alpha}\right] \cap P_{\alpha}=\varnothing$ and $\left|P_{\alpha}\right|=|\alpha|$ if $\alpha \geqslant \omega$ and $\left|P_{\alpha}\right|<\omega$ if $\alpha<\omega$. $\left|\cup_{\alpha<\beta} P_{\alpha}\right|=\cup_{\alpha<\beta}|\alpha|=|\beta|<$ $2^{\mathrm{c}}$ if $\alpha \geqslant \omega$ and is less than $\omega$ if $\alpha<\omega$. Consider $K_{\beta}$. Several cases arise:

(i) $\exists r_{\beta}$ such that $K_{\beta} \cap\left(\left\{r_{\beta}\right\} \times \beta N\right)$ is infinite.

(a) $r_{\beta} \in f\left(\cup_{\alpha<\beta} P_{\alpha}\right) \subset \beta N-N$. Let $P_{\beta}=\cup_{\alpha<\beta} P_{\alpha}$. In this case, $P$ will be defined so that $K_{\beta} \not \subset P$ hence $K_{\beta}$ need not have a limit point in $X^{2}$.

(b) $r_{\beta} \notin f\left[\cup_{\alpha<\beta} P_{\alpha}\right]$. Choose $s_{\beta} \in \beta N-\left(f\left[\cup_{\alpha<\beta} P_{\alpha}\right] \cup N\right)$ so that $\left(r_{\beta}, s_{\beta}\right) \in \operatorname{cl} K_{\beta}-\left(G \cup K_{\beta}\right)$. Let $P_{\beta}=\cup_{\alpha<\beta} P_{\alpha} \cup\left(\left\{r_{\beta}, s_{\beta}\right\}-N\right)$.

(ii) We have an analogous case if $\exists s_{\beta}$ such that $K_{\beta} \cap\left(\beta N \times\left\{s_{\beta}\right\}\right)$ is infinite.

(iii) If no such points exist, apply the lemma, using a simple cardinality argument, to obtain a point $\left(r_{\beta}, s_{\beta}\right)$ so that $r_{\beta}, s_{\beta} \notin f\left[\cup_{\alpha<\beta} P_{\alpha}\right] \cup N$ and $\left(r_{\beta}, s_{\beta}\right) \in \operatorname{cl} K_{\beta}-\left(G \cup K_{\beta}\right)$. Let $P_{\beta}=\cup_{\alpha<\beta} P_{\alpha} \cup\left\{r_{\beta}, s_{\beta}\right\}$.

So clearly, $\left|P_{\beta}\right|=\left|\cup_{\alpha<\beta} P_{\alpha}\right|=|\beta|$ if $\alpha \geqslant \omega$ and is finite otherwise. Equally clear is that $P_{\beta} \supset P_{\alpha}$ for $\alpha<\beta$.

Claim. $f\left[P_{\beta}\right] \cap P_{\beta}=\varnothing$. Let $p \in P_{\beta}$ and suppose $\exists q \in P_{\beta}$ such that $f(q)=p$. Note the following:

(i) Obviously, the inductive hypothesis guarantees that not both $p, q \in$ $\bigcup_{\alpha \in \beta} P_{\alpha}$.

(ii) If $p \in \cup_{\alpha<\beta} P_{\alpha}$ and $q=r_{\beta}$, we have $f\left(r_{\beta}\right)=p$. So $f(p)=r_{\beta}$. But $r_{\beta}$ was chosen so that $r_{\beta} \notin f\left[\cup_{\alpha<\beta} P_{\alpha}\right]$.

(iii) If $p \in \cup_{\alpha<\beta} P_{\alpha}$ and $q=s_{\beta}, f\left(s_{\beta}\right)=p$, so $f(p)=s_{\beta}$ and we have a contradiction as above.

(iv) If $p=r_{\beta}$ and $q=s_{\beta}$, we have $f\left(s_{\beta}\right)=r_{\beta}$ so that $f\left(r_{\beta}\right)=s_{\beta}$. But this gives $\left(r_{\beta}, s_{\beta}\right) \in G$, a contradiction.

(v) If $p=s_{\beta}$ and $q=r_{\beta}, f\left(r_{\beta}\right)=s_{\beta}$, again a contradiction.

The claim now follows.

The inductive construction of the example is now complete. Note that $P^{2}$ is countably compact.

REMARKS. (1) The example presented here is a partial negative answer to a question of $\mathbf{J}$. Keesling, whose interest in the problem stems from research announced in $[\mathrm{K}]$ concerning hyperspaces. The question, to which I do not know the answer, is: If $X$ is normal and countably compact and $X^{2}$ is countably paracompact, is $X^{2}$ countably compact? R. G. Woods [Wo] has shown that $\mathrm{CH}$ implies that if $X$ is normal, countably compact, extremally disconnected and $\left|C^{*}(X)\right|=2^{\omega}$, then $X$ is compact. Thus the present example is not normal assuming $\mathrm{CH}$.

(2) The example presented here also answers in the negative the following question of Morita [M]: If $X$ and $Y$ are countably compact and $X \times Y$ is an $M$-space, is $X \times Y$ countably compact? The question had been answered in the negative by Steiner [S], assuming the continuum hypothesis. An $M$-space is the quasi-perfect preimage of a metric space. Note that $X^{2}$ is an $M$-space: It is the free union of a countably compact space and a countably infinite discrete space. See also [Wa, pp. 188-190]. 
(3) An example, due to Frolík, of countably compact spaces $X$ and $Y$ whose product is pseudocompact but not countably compact is presented by Ginsburg and Saks in [GS]. Only slight modification is needed to yield a countably compact space whose square is pseudocompact but not countably compact. Similar results can be obtained from the example given by Comfort in [C].

\section{REFERENCES}

[C] W. W. Comfort, A nompseudocompact product space whose finite subproducts are pseudocompact, Math. Ann. 170 (1967), 41-44. MR 35 \#965.

[E] R. Engleking, Outline of general topology, Wiley, New York, 1968. MR 37 \#5836.

[F] Z. Frolik, Generalizations of compact and Lindelöf spaces, Czechoslovak Math. J. 9 (84) (1959), 172-217. MR 21 \#3821.

[GJ] L. Gillman and M. Jerison, rings of continuous functions, Van Nostrand, Princeton, N. J., 1960. MR 22 \#6994.

[GS] J. Ginsberg and V. Saks, Some applications of ultrafilters in topology, Pacific J. Math. 57 (1975), 403-418. MR 52 \# 1633.

[K] J. Keesling, Normality and compactness are equivalent in hyperspaces, Bull. Amer. Math. Soc. 76 (1970), 618-619. MR 40 \#8019.

[M] K. Morita, A survey of the theory of M-spaces, General Topology and Appl. 1 (1971), 49-55. MR 44 \#3276.

[N] J. Novak, On the Cartesian product of two compact spaces, Fund. Math. 40 (1953), 106-112. MR 15, 640.

[S] A. K. Steiner, On the topological completion of $M$-space products, Proc. Amer. Math. Soc. 29 (1971), 617-620. MR 43 \#8051.

[SS] L. A. Steen and J. A. Seebach, Jr., Counterexamples in topoloyy, Holt, Rinehart and Winston, New York, 1970. MR 42 \# 1040.

[T] H. Terasaka, On the Cartesian product of two compact spaces, Osaka J. Math. 4 (1952), 11-15. MR 14, 489.

[Wa] R. C. Walker, The Stone-C̈ech compactification, Springer-Verlag, Berlin and New York, 1974. MR 52 \# 1595.

[Wo] R. G. Woods, The structure of small normal F-spaces, Proc. Auburn Conf. (Auburn Univ., Ala., March, 1976) (to appear).

Departmient of Mathematics, Allegheny College, Meadville, Pennsylvania 16335 